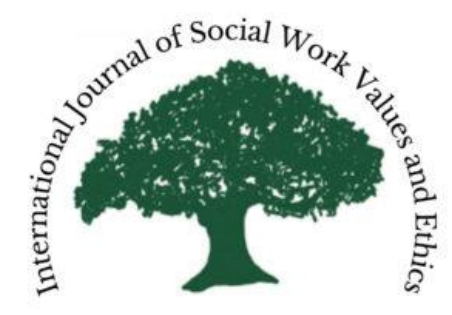

International Journal of Social Work Values and Ethics

Volume 19, Number 1 (2022)

ISSN: 2790-6345

DOl: 10.55521/10-019-100

www.jswve.org 


\section{Some Notes on the Journal's New Features}

DOI: $10.55521 / 10-019-103$

Pascal Rudin, Ph.D., Publisher

\section{International Journal of Social Work Values and Ethics • Volume 19(1), Copyright 2022 by IFSW}

This text may be freely shared among individuals, but it may not be republished in any medium without express written consent from the authors and advance notification of IFSW.

With IFSW being the new publisher, the name of the Journal has now been changed to International Journal of Social Work Values (i.e., the word "International" has been added) to reflect the increasingly global authorship and audience. This is also reflected in the new ISSN number 2790-6345 which has been linked to the former publication titled Journal of Social Work Values.

Against this backdrop, a number of new exciting features have been introduced. These include:

\section{New Layout}

The Journal comes in a completely new, modern and sleek design. In consultation with an external layout expert a number of optimisations have been introduced. These include:

- the format has been changed from $\mathrm{A} 4$ to $\mathrm{A} 5$, to optimise it for ereaders, tablets and even smartphones. With mobile devices now being the majority of Internet devices used across the globe, we wanted to optimise the Journal's design accordingly. This trend is further accounted for by HTML text that is fully responsive (i.e. it adapts to the screen size of any device).

- the colour palette has been derived from the well-established logo and website to ensure continuity. 
- modern Google fonts have been used to optimise readability: Oswald font for titles and descriptions, as well as Alegreya Sans font for content.

- the Journal now comes in a one-column design to optimise both readability and accessibility.

\section{DOI Identifiers}

IFSW now assigns a digital object identifier (DOI) to all articles and book reviews published by the Journal as soon as the content is made available electronically. This is a unique numeric string to identify content and provide a persistent link to its location on the Internet (i.e. the Journal's website). In order to achieve this, IFSW became a member of CrossRef, where more than $80 \%$ of the global citation meta data of DOI indexed content are stored. Through DOIs, articles can more easily be identified both by search engines and databases.

IFSW's membership with Crossref increases the authenticity of this Journal and reflects the growing impetus for an international culture of citation using the DOI system. This approach increases acceptance of research data as legitimate and citable contributions to the scholarly record, which is important for both the Journal and authors publishing with us.

\section{HTML Full Text}

One of the requirements of the $\mathrm{DOI}$ system is to have a landing page for each content (e.g. article, book review). This page needs to carry the citation meta data of the relevant content item, such as author, date, file location etc. We go one step further and also publish the full HTML text of the relevant content item. This allows users to read articles without even accessing the relevant PDF. For smaller screens, such as smartphones, content will be better accessible due to the responsiveness of the Journal's website (i.e. the text will display differently in response to the screen size to optimise readability). 


\section{Automatic Translation}

A further advantage of having full HTML content published is the ability to translate text into one's own language. Website visitors may choose the newly installed Google translate functionality to read content in their own language. While the quality of these translations has been improved over the past years and continues to improve, it remains an automatic translation with its specific limitations. Nevertheless, this functionality may help our increasingly global audience to read articles and book reviews in their own language.

\section{Full Citation Meta Data}

As mentioned before, each article and book review will come with its own landing page that also carries full citation meta data. Through this, website visitors may now use citation software such as EndNote, Mendeley or Zotero to download not only the full citation of a specific article or book review, but also the PDF completely automatically into their library at the ease of a click. No manual writing down of authors, date, Journal name etc. required anymore.

IFSW is delighted to present all these exciting features to our global audience and wishes everyone an interesting read! 